\title{
POSIBLES INFLUENCIAS \\ DE LA MIGRACION EN LOS CAMBIOS DE LA FECUNDIDAD
}

(A partir de un estudio de la ciudad de Barcelona, 1970-1981)

\section{Graciela Sarrible}

\section{INTRODUCCION}

La fecundidad de las mujeres migrantes no sólo ha constituido un tema de interés para los especialistas en el estudio de la población, sino que ha sido motivo de controversia. En general, existen coincidencias al señalar el carácter diferencial de la fecundidad de las personas móviles - respecto de las no-móviles, tanto en origen como en destino-. El desacuerdo consiste en considerar que los niveles son superiores o inferiores, sobre todo respecto de la sociedad de llegada ${ }^{1}$.

Estos aparentes desacuerdos se deben a varias razones. Entre ellas podría apuntar las señaladas por varios autores, a saber:

$1 .^{\mathrm{a}}$ Diferencias fundamentalmente metodológicas y la utilización de distintos indicadores para medir la fecundidad ${ }^{2}$.

2. ${ }^{a} \quad$ Diferencias en el contexto histórico y en los tipos de migración ${ }^{3}$.

1 Entre los que consideran que la fecundidad de las migrantes hacia las áreas urbanas es superior podemos citar a Myers, Martine, Guillon. De los partidarios de la posición contraria mencionaremos a Hiday, Rindfuss, Courgeau.

${ }_{2}^{2}$ ZÁRATE y UNGER DE ZÁRATE, «On the Reconciliation of research Findings of Migrants-Non-Migrants Fertility Differentials in Urban Areas», The International Migration Review, vol. IX, núm. 2, 1975.

${ }^{3}$ G. SABAg H y Sun BIN YIN, "The Relationship between Migration and Fertility in 
3. Diferencias entre las distintas etnias que forman parte de la población migrante 4 .

4. ${ }^{2}$ Diferencias en la composición socioeconómica de ambas subpoblaciones ${ }^{5}$.

5. ${ }^{\mathrm{a}}$ Diferencias entre nuevas y más antiguas migraciones ${ }^{6}$.

Las razones antes apuntadas no son excluyentes. De hecho, algunos autores consideran simultáneamente varias de ellas al describir el contexto donde se perciben esas diferencias de fecundidad. Destaca, sin embargo, la diversidad de situaciones, momentos históricos y sociedades donde las investigaciones se han llevado a cabo. Es de esperar, entonces, que los resultados sean tan dispares. La uniformidad de comportamiento sería difícil de encontrar. Pero muchas de estas formulaciones han servido de base para intentar explicar las distintas situaciones que han ido surgiendo al analizar la fecundidad de nativas y migrantes en la ciudad de Barcelona, en la década del $70^{7}$.

Esta investigación se ha centrado en el estudio comparativo de la comunidad catalana y migrante en el municipio de Barcelona, en términos de fecundidad. Existe una pluralidad de factores demográficos y sociales que diferencia ambas subpoblaciones. El objetivo de este artículo es el de visualizar los comportamientos distintivos, así como mostrar la similitud entre las nuevas generaciones.

En un tiempo tan corto - sólo una década estudiada en profundidadlos cambios se han sucedido de manera vertiginosa. En ningún caso se ha pretendido encuadrar las transformaciones operadas en la comunidad migrante, como si de un proceso de aculturación o de asimilación se tratara. Me he limitado a constatarlos y a intentar indagar las razones que los han motivado.

La fecundidad de la ciudad ha evolucionado desde un incremento -en el primer quinquenio- a un descenso que aún hoy no se ha detenido. En esta atmósfera de cambios profundos se ha supuesto - y tratado de demostrar- que las dos subpoblaciones estudiadas han reaccionado de manera distinta, pero que, globalmente, las mayores diferencias son observables entre las nuevas y más antiguas generaciones, más allá del origen de las mujeres que formaban parte de ellas. Las más jóvenes, entonces, serían las promotoras de estos cambios en la fecundidad, independientemente de su procedencia,

an Historical Context: The Case of Morocco in 1960», The International Migration Review, vol. XIV, núm. 52, 1980.

${ }^{4}$ Avery M. Guest, «Fertility Variation Among the US Foreign Stock Population in 1900», The International Migration Review, vol. XVI, núm. 3, 1982.

${ }_{5}$ P. A. Compton, «Fertility Differentials and their Impact on Population Distribution and Composition in Northern Ireland», Environment and Planning A., Dec. 1978, Northern Ireland.

6 V. Hiday, «Migration, Urbanization and Fertility in the Philippines», The International Migration Review, vol. XII, núm. 3, 1978.

7 Nota: A efectos de este trabajo, consideraremos como no-móviles a las mujeres que residen en su Comunidad Autónoma de origen, o sea, Cataluña, y como migrantes a las nacidas en el resto de Comunidades Autónomas de España. 
que evidenciaría diferencias de menor cuantía. En las más antiguas generaciones, sin embargo, los comportamientos diferenciados —por origen o por categoría profesional- resultarían más contrastados.

La comunidad migrante no constituye un todo homogéneo. La desagregación por Comunidad Autónoma de origen sería necesaria si quisiéramos insistir en las diferentes pautas o respuestas de estos colectivos de mujeres ${ }^{8}$. La distinción fundamental la constituirá el hecho de haber realizado o no desplazamientos que las lleven a residir fuera de su comunidad de nacimiento. La migración constituiría un hito en sus historias de vida. De ahí que se intentará deslindar en qué medida ha alterado su fecundidad.

\section{PROBLEMAS DE DEFINICION DE VARIABLES}

\section{La fecundidad urbana}

Para poder hablar de la fecundidad diferencial de los residentes en una ciudad es necesario que precisemos el concepto de fecundidad urbana. Se entiende por «fecundidad urbana» la fecundidad de las mujeres que residen, permanentemente, en la ciudad. Aun cuando parezca una reiteración, esta definición constituye sólo una de las posibles. Por ejemplo, se podría haber definido como fecundidad de Barcelona-ciudad la de las mujeres nacidas y residentes en ella. Esto hubiera implicado eliminar a todas las demás - nacidas fuera-, sin importar los años que tuvieran de residencia. Evidentemente, cuando se comenta el incremento de la fecundidad de las ciudades siempre se tiene en cuenta los nacimientos allí ocurridos, sin distinguir dónde han nacido las mujeres (que pueden haber nacido en esa o en otras ciudades o en zonas rurales).

La fecundidad, tal como la definimos, tiene la ventaja de representar la media ponderada de los distintos subgrupos y subpoblaciones que viven en la ciudad. Además, desde este punto de vista adoptado, la fecundidad de las mujeres migrantes se estudia como un hecho interno y su aporte particular está incluido en la media general de la ciudad. Tenemos así dos grupos, al menos, con los que podamos comparar: el de las nativas y el de las no-móviles.

Cabe decir, por último, que es la solución más corriente, ya que en general no necesita una reelaboración de los datos y es adoptada por ello sin necesidad de explicitar exactamente su contenido.

Debido al aporte de las migrantes, que se concentran en nuestro caso y en otros en las edades fecundas, es usual que se tienda a sobreestimar lo que sería el número medio de hijos de esas mujeres. Si bien la tasa de natalidad está influida por la estructura, la tasa de fecundidad global lo está en menor

${ }^{8}$ Las características diferenciales por Comunidad Autónoma serán motivo de otro artículo, ya que, dada la extensión del trabajo, no podrían ser incluidas en el presente. 
grado, pero también depende de la composición y peso de los distintos grupos de edades y su repartición en las edades más o menos fecundas.

Por ejemplo, en un artículo de Hémery y Rabut, el índice transversal de fecundidad de las mujeres españolas residentes en Francia alcanzaba 3,2 hijos en $1968^{\circ}$, mientras que en 1965-66 había sido estimado por Sáez en 2,94 $y$ en $1970-71$ en 2,88 , para toda España ${ }^{10}$.

Por supuesto, dicho artículo insiste en que las extranjeras tienen más hijos porque reproducen una fecundidad superior de origen, sin tener en cuenta, como en la mayoría de los casos, que la fecundidad en el sitio de origen resulta bien diferente.

Dadas las migraciones del campo a la ciudad, muy importantes estos últimos años, y, en general, las migraciones hacia las grandes aglomeraciones desde todos los municipios, pequeños y medianos, las tasas de natalidad de las grandes ciudades, como Barcelona, se han visto incrementadas. Al mismo tiempo, las descendencias -medidas por la pregunta censal- se aproximaban entre la provincia y la capital (en Barcelona) ${ }^{11}$.

La diferencia, sin embargo, es que los cambios en Barcelona han sido muy rápidos. De una fecundidad elevada en los años setenta — sobre todo el primer quinquenio-, la ciudad ha pasado a una fecundidad relativamente reducida.

Si Andorka considera que las fluctuaciones económicas afectan los indicadores transversales de fecundidad ${ }^{12}$, se puede considerar que la influencia ha de ser mayor en las ciudades. De ahí los cambios fundamentales que ha vivido Barcelona, en un período relativamente tan corto.

\section{La población migrante}

El problema de la migración implica una toma de posición al definir al migrante.

De acuerdo con los datos y con todos los presupuestos teóricos ya expuestos, nuestra acepción no podría ser otra que la de haber nacido fuera de la comunidad de residencia, o sea, Cataluña. Ello implica dejar de lado el problema de migraciones múltiples o de tiempos de permanencia. Además, las hijas de las migrantes nacidas en Cataluña son consideradas igualmente que las catalanas de varias generaciones. Obviamente, se trata de una simplificación, puesto que si el cambio no deja de ser un fenómeno generacional, no es evi-

9 S. HÉmery y O. RABUT, «La contribution des étrangers à la natalité en France», Population, vol. 28, núm. 6, INED, París, 1973.

${ }^{10}$ A. SÁEz, «La fecondité en Espagne depuis le début du siècle», Population, núm. 6, INED, París, 1979.

"Nota: Según el Censo de 1970, el número medio de hijos en Barcelona era: provincia, 2,26; zona urbana, 2,25, y capital, 2,12 .

${ }_{12}$ R. Andorka, Determinats of fertility in Advanced Societies, Londres, Methuen, 1978. 
dente que la segunda generación tenga igual comportamientos que las autóctonas de varias generaciones.

Pero no podemos olvidar, en defensa de la opción elegida, que la tendencia a la homogeneización que existe contribuirá a que las variaciones sean tan pequeñas que perderán importancia en el futuro y no resultarán significativas.

\section{LA COMUNIDAD CATALANA}

\section{Natalidad y fecundidad global}

Los nacimientos de las mujeres cuyo lugar de origen es Cataluña presentan series regulares. Sin embargo, si distinguimos las nacidas en la ciudad, las del resto de la provincia y las del resto de Cataluña, podemos encontrarnos con algunas alteraciones. En 1981, la proporción respecto del total de nacimientos de la ciudad se reduce y, al mismo tiempo, aumentan los «no-consta». Por eso hemos optado por reunir Barcelona-ciudad y provincia, ante la inseguridad que supone una redistribución de los nacimientos de la categoría «no-consta».

Los nacimientos atribuidos a madres cuyo origen es la ciudad de Barcelona registran una tendencia general similar a la del total de la ciudad, pero la magnitud de sus cambios resulta bien diferente. Tanto sus incrementos como sus descensos son de menor cuantía. Los nacimientos correspondientes a madres de la provincia presentan oscilaciones continuas, pero en términos generales aumentan entre 1970 y 1983; al contratio de lo que sucede con los del resto de Cataluña, que descienden más que la media de la ciudad (cuadro I).

Debido a estas tendencias contrapuestas entre sus componentes, el total de nacimientos correspondientes a madres de origen catalán resultan más regulares. O sea, que, relativamente, la natalidad de las catalanas presenta menos oscilaciones, en el período analizado, que la ciudad en su conjunto.

La contribución de las mujeres nacidas en la ciudad de Barcelona a los nacimientos ilegítimos es la que más ha aumentado en este período, mientras que la correspondiente a mujeres del resto de la provincia y de las restantes provincias catalanas se ha mantenido cuasi-estable y su aporte continúa siendo reducido al final del período. El total de madres catalanas que han dado lugar a un nacimiento ilegítimo depende, casi exclusivamente, del aporte de las mujeres nacidas en la ciudad. Su máximo (1982) y su mínimo (1978) corresponden a los respectivos máximo y mínimo de las mujeres originarias de la capital. 


\section{CUADRO I}

Evolución general de los nacimientos según naturaleza de la madre: numeros indices, 1970-1984

(Año base 1970: 100)

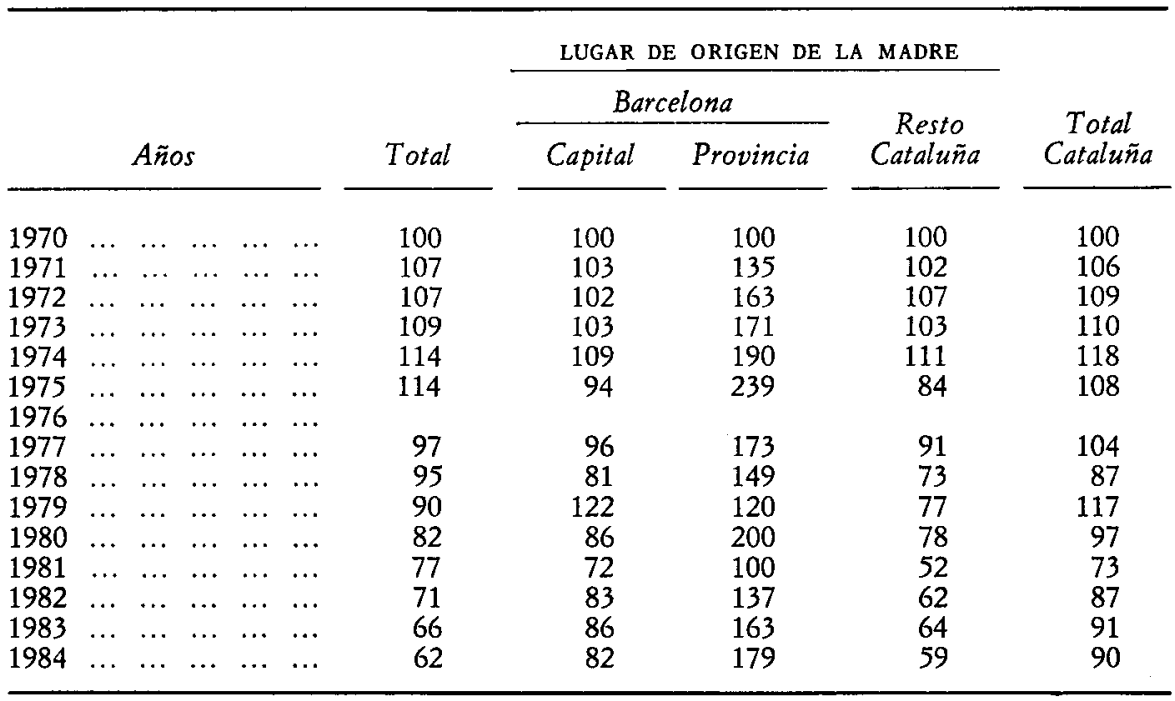

Fuentes: Estadística Municipal, años 1970 a 1984.

Elaboración propia.

Este aumento absoluto es de mucha menor cuantía en términos relativos, puesto que no alcanza el 1 por 100 de los nacimientos totales.

Las tasas de fecundidad global correspondientes a 1970 para las mujeres nacidas en Barcelona y en el resto de Cataluña resultan casi idénticas ( 77 por 1.000 ambas, con diferencias sólo en los decimales). Sin embargo, las notables diferencias entre mujeres nacidas en la capital $(66,26$ por 1.000$)$ y las de la provincia $(203,58$ por 1.000$)$ nos ha obligado, en 1975 , a construir una sola tasa (que resulta de 78 por 1.000). Estas notables variaciones en las magnitudes pueden deberse a que la hipótesis de equirrepartición (de los nacimientos de vecinos y transeúntes) no se verifique para las madres de la provincia - contadas aparte-. Ello implicaría que una proporción superior de nacimientos de estas madres corresponden a transeúntes, por encima de la media general de la ciudad ${ }^{13}$.

${ }^{13}$ Por la hipótesis de equirrepartición se aplica la distribución de vecinos y transeúntes observada para el conjunto de la ciudad a las distintas comunidades estudiadas. 
Las tasas correspondientes al resto de Cataluña y al total de Cataluña continúan siendo similares a las de las mujeres de Barcelona-provincia -incluida la capital- (cuadro II).

\section{CUADRO II}

Tasa media global de fecundidad por lugar de origen (Media de la tasa de 70-71, 75-76 y 80-81, ciudad de Barcelona)

\begin{tabular}{|c|c|}
\hline Lugar de origen & Tasa media (Tx) \\
\hline $\begin{array}{l}\text { Capital y provincia de Barcelona } \ldots \ldots \ldots \\
\text { Resto de Cataluña }\end{array} \ldots$ & $\begin{array}{r}106,25(1) \\
76,80(1)\end{array}$ \\
\hline Total Cataluña $\ldots \ldots \ldots \ldots$ & 71,85 \\
\hline Total España $(\sin$ Cataluña) $\ldots \ldots \ldots$ & 134,19 \\
\hline 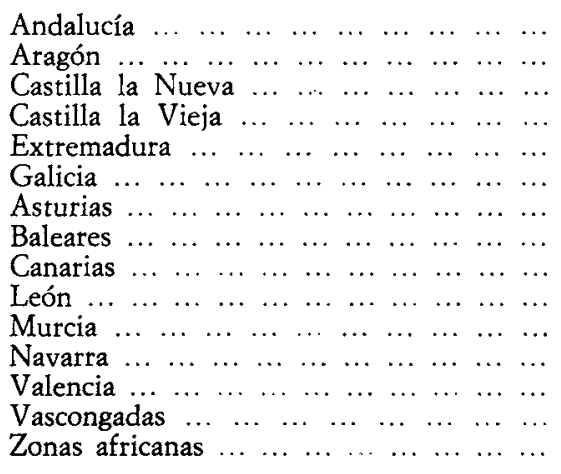 & $\begin{array}{l}179,42 \\
54,37 \\
139,10(1) \\
87,30(1) \\
209,40 \\
91,60 \\
70,62 \\
75,15 \\
119,12(2) \\
109,46(1) \\
119,85(1) \\
69,35 \\
84,60 \\
66,14(2) \\
-\end{array}$ \\
\hline Total $(\sin$ Ext., $\sin \mathrm{NC}) \ldots \ldots \ldots$ & 96,56 \\
\hline $\begin{array}{lllllllll}\text { Total } & \ldots & \ldots & \ldots & \ldots & \ldots & \ldots & \ldots & \ldots\end{array}$ & 103,84 \\
\hline
\end{tabular}

(1) La tasa media ha sido construida sólo a partir de las tasas de $70-71$ y 75-76, por no disponer de las últimas.

(2) La tasa media ha sido construida sólo a partir de las tasas de $70-71$ y $80-81$, por considerarse sobrevalorada la del 75-76.

Elaboración propia.

Para 1981 sólo hemos podido calcular una única tasa. Su valor resulta muy inferior a los dos anteriores. O sea, que la fecundidad de las catalanas, medida por su tasa global, resulta casi idéntica entre 1970-71 y 1975-77 $(77,55$ y 77,85 por 1.000 , respectivamente), pero muestra un marcado descenso al final del período, ya que se sitúa en 60,15 por 1.000 .

El incremento registrado en la media de la ciudad en torno a la mitad del quinquenio no se debe a esta comunidad, que presenta niveles uniformes respecto al inicio, sino a la de migrantes. 


\section{Solteria y nupcialidad}

Coincidiendo con una menor fecundidad, las mujeres catalanas presentan una elevada proporción de solteras, mayor que la del total de la ciudad y, por supuesto, que la de mujeres nacidas en el resto de España - en su conjunto- Globalmente, casi la mitad de las catalanas en edad fecunda están solteras, en 1981 ( 46 por 100). Las diferencias con las medias de la ciudad son reducidas a partir de los 30 años, pero son muy importantes antes de esa edad, cuando las tasas de fecundidad son elevadas. Sin embargo, las catalanas se están casando más que antes, ya que la soltería definitiva - que podemos considerar a partir de las proporciones del último grupo de edad (45-49 años)resulta superior a la observada en los dos grupos de edad anteriores (35-39 y 40-44 años). A pesar de ello, las catalanas se siguen casando más tarde y menos que las mujeres de España, que el conjunto de mujeres residentes en Barcelona y, lógicamente, que el conjunto de mujeres residentes en Barcelona pero nacidas en el resto de comunidades de España. Ello no implica que su soltería sea superior a la de cualquier comunidad española, sino solamente respecto a la media de las migrantes.

Si consideramos todas las mujeres residentes en Cataluña, como referencia, observaremos que se han casado mucho más que las residentes en la ciudad. Las diferencias son notables, al punto que la soltería a partir de los 40 años es de casi el doble en la ciudad que en el conjunto de Cataluña.

Siempre se ha insistido en el efecto negativo de la urbanización en la fecundidad de las mujeres, pero rara vez se tiene en cuenta un hecho anterior y decisivo, como es la soltería. En este caso, pese a que las probabilidades estadísticas de un mercado matrimonial extenso serían superiores a las de los más pequeños municipios, de hecho, la frecuencia de la soltería es mayor.

\section{Número medio de bijos}

Sin embargo, la descendencia media de las mujeres alguna vez casadas, medida en 1977 por la Encuesta Nacional de Fecundidad, da unas proporciones superiores a las mujeres residentes en Madrid (una media de 2,13 hijos) que a las de Barcelona (2,05). Lo mismo ocurre en la Encuesta de Fecundidad de 1985, que en su avance de resultados presenta una media de la Comunidad Autónoma de Madrid de 2,26 hijos, actualmente vivos — de mujeres que han tenido al menos un nacido vivo- ${ }^{14}$, y de 2,21 para Cataluña ${ }^{15}$. En cambio, en la media de hijos del colectivo total la relación se invierte, siendo superior

${ }^{14}$ Cuadros 3.6.1 y 3.29.1 de la Encuesta Nacional de Fecundidad, Avance de Resultados, INE, Madrid, 1986.

${ }^{15}$ Las ciudades de Madrid y Barcelona no constituyen unidades de análisis en esta nueva encuesta. 
la de Cataluña (con 1,50) respecto de la Comunidad Autónoma de Madrid $(1,45)$.

La media de hijos más elevada de los distritos de Barcelona (en 1981) coincide con la mayor proporción de catalanas. Estas mujeres tendrían una fecundidad más tardía y, por ende, un calendario más concentrado, ya que la intensidad final resulta superior a la de los restantes distritos y a la del conjunto de la ciudad. Pero cuando las proporciones entre migrantes y catalanas son similares, y los distritos corresponden fundamentalmente al asentamiento de clases medias, la fecundidad resulta la más baja de todas.

\section{La pirámide de edades}

La proporción de mujeres catalanas en edad fecunda crece, tanto en 1975 como en 1981, respecto al total de mujeres comprendidas entre esas edades. Esto se debe, fundamentalmente, al aumento de mujeres nacidas en Barcelonaciudad (cuadro III).

Entre 1970 y 1975 , los efectivos de mujeres originarias de Barcelona se incrementan - si consideramos los mismos grupos de edad- hasta los 34 años y en el último grupo; o sea, que aumentan los grupos más fecundos. Mientras que las mujeres del resto de Cataluña reducen sus efectivos, en todos los grupos de edad. Sin embargo, este aumento es exclusivamente debido a un efecto "generación», o sea, a la diferencia de efectivos que entran y avanzan en edad, respecto de los más viejos. Si seguimos las generaciones (efectivos en 1970

\section{CUADRO III}

Estructura de la población catalana residente en Barcelona, mujeres de $15-49$ años, en 1970, 1975 y 1981

\begin{tabular}{|c|c|c|c|c|c|c|c|}
\hline \multirow{2}{*}{$\begin{array}{l}\text { Grupos } \\
\text { de edad }\end{array}$} & \multicolumn{2}{|c|}{ Barcelona } & \multicolumn{2}{|c|}{ Resto de Cataluña } & \multicolumn{3}{|c|}{ Total Cataluña } \\
\hline & 1970 & 1975 & 1970 & 1975 & 1970 & 1975 & 1981 \\
\hline $15-19$ & 38.287 & 46.311 & 2.230 & 1.378 & 40.517 & 47.689 & 56.146 \\
\hline $20-24$ & 32.723 & 35.382 & 3.130 & 2.586 & 35.853 & 37.968 & 44.583 \\
\hline $25-29$ & 26.151 & 30.844 & 3.079 & 3.400 & 29.230 & 34.244 & 32.433 \\
\hline $30-34 \ldots \ldots \ldots$ & 23.110 & 25.097 & 2.786 & 3.170 & 25.896 & 28.267 & 31.263 \\
\hline $35-39$ & 27.944 & 22.016 & 3.695 & 2.767 & 31.639 & 24.783 & 27.250 \\
\hline $40-44 \ldots \ldots \ldots$ & 29.586 & 26.597 & 4.240 & 3.646 & 33.826 & 30.243 & 23.393 \\
\hline $45-49 \ldots \ldots \ldots$ & 27.725 & 28.138 & 4.943 & 4.203 & 32.658 & 32.341 & 29.362 \\
\hline Total . ... & 205.516 & 214.385 & 24.103 & 21.150 & 229.619 & 235.535 & 244.430 \\
\hline
\end{tabular}

Fuentes: Censos y Padrones de 1970, 1975 y 1981. 
y 1975) observaremos que disminuyen. O sea, que, a pesar del incremento absoluto, se percibe una emigración, fuera de los límites de la ciudad, de las mujeres nacidas en Barcelona. Mientras las mujeres del resto de Cataluña —que pierden efectivos globalmente- reciben aportes positivos de migrantes en los tres primeros grupos de edad, correspondientes a 1970, o sea, un colectivo de quince generaciones.

El total de la población catalana sigue las tendencias de la población barcelonesa, que es mayoritaria; incrementa sus efectivos absolutos, tanto en 1975 como en 1981, en los cuatro primeros grupos de edad, hasta los 34 años, y pierde en los tres últimos. Si observamos las generaciones, a partir de 1970 los efectivos se reducen en todos los casos.

En síntesis, se combina un aumento de la población, sobre todo en las edades más fecundas, por efecto del avance en edad de generaciones más numerosas, pero las pérdidas por emigración son constantes en todos los casos, y más importantes en los primeros grupos de edad que en los últimos.

El incremento absoluto se produce porque las generaciones más recientes están compuestas por catalanas de segunda generación e hijas de migrantes. O sea, que la pirámide se ha rejuvenecido en la base, por efecto de la fecundidad pasada y, sobre todo, por el aporte de la migración al crecimiento natural.

La pérdida de efectivos, o sea, la emigración, se puede producir por la falta de posibilidades de las parejas jóvenes, de menos recursos, de continuar habitando Barcelona-ciudad, como sus padres, trasladándose a ciudades vecinas. Debemos de tener en cuenta que el ámbito espacial que define la migración es exclusivamente municipal.

Considerando la estructura, en términos de fecundidad, diremos que el conjunto de mujeres catalanas en edad fecunda tiene cada vez una distribución más favorable, tanto en 1975 como en 1981; aunque sólo en la última fecha resulta, comparativamente, más favorable que la media de la ciudad.

Las estructuras barcelonesas y de mujeres nacidas en el resto de Cataluña también resultan más favorables en 1975 (la primera más que la segunda), aunque en esas fechas todavía están por debajo de la media de la ciudad.

En sintesis, separando los factores positivos o negativos respecto de la fecundidad, tenemos:

- Positivos, o favorables a la fecundidad:

- Incrementos de los nacimientos absolutos, totales e ilegítimos, y descensos, relativamente, de menor cuantía.

- Distribución más favorable a la fecundidad en 1975 y 1981, aunque sólo en la última fecha supera la media de la ciudad.

- Incremento absoluto de efectivos, sobre todo en las edades más fecundas. 
- Migraciones positivas, pero muy reducidas, del resto de Cataluña hacia la ciudad, en el primer quinquenio.

- Negativos, o desfavorables a la fecundidad:

- Emigración constante durante el período de mujeres nacidas en Barcelona.

- Proporciones de solteras muy elevadas, sobre todo en edades fecundas (aunque la soltería definitiva no presenta tantas diferencias, lo que implicaría casamientos más tardíos que el conjunto).

- Distribuciones en 1970 y 1975 menos favorables que la media de la ciudad.

Como balance final, se resumen las características de la fecundidad de las catalanas residentes en la ciudad y se intentará explicar las razones del descenso:

Primera. La fecundidad de las catalanas resultó mucho más uniforme en un período de diez años; o sea, que los cambios de las mujeres definidas como no-móviles resultan más lentos, o, si se quiere, su fecundidad está menos alterada, analizada transversalmente.

Segunda. Sólo se pueden definir dos componentes de la comunidad catalana, las nacidas en la provincia de Barcelona -incluida capital- y las nacidas en las tres provincias restantes. De otro modo, la hipótesis de equirrepartición de nacimientos vecinos y transeúntes resultaría improbable (si se diferencian a las nacidas en la capital y en el resto de la provincia).

Tercera. La situación por estado civil de las catalanas resulta desfavorable, al menos a una fecundidad legítima, que sigue siendo mayoritaria. Cuando se habla del efecto negativo de las grandes ciudades respecto de la fecundidad debería tenerse en cuenta que el efecto es negativo, primero, en términos de fecundidad general y, luego, se debería analizar su influencia en la fecundidad legítima. Según el Censo de 1970, las diferencias entre zona urbana y rural, en Barcelona, son mínimas. Según la Encuesta de 1977, la descendencia de mujeres alguna vez casadas crece regularmente en Cataluña con el tamaño del municipio, salvo el caso de Barcelona-ciudad; mientras que en España no sucede lo mismo. O sea, que en Barcelona, y quizá también en Cataluña, no existiría una correlación clara, unívoca o positiva entre urbanización y descenso de la fecundidad.

Cuarta.-Si bien la distribución de las mujeres entre 15 y 49 años se va haciendo más favorable a la fecundidad, según avanzamos en años calendarios era-menor a la media de la ciudad en el momento de mayor fecundidad global, y sólo resulta más favorable cuando ya se ha concretado el descenso. Los efectivos de mujeres catalanas se mueven en sentido inverso que el total de 
la ciudad $\mathrm{y}$, por ende, de la comunidad migrante. Son los únicos que crecen en términos absolutos, aunque la salida de migrantes es continua, sobre todo en las generaciones más jóvenes.

Quinta. Por todo ello, podríamos esperar una fecundidad menor que la media, al menos hasta 1975-76 (en que las condiciones de las catalanas eran más desfavorables que el conjunto y, por ende, que la comunidad migrante). Pero cuando sus condiciones mejoran el descenso resulta inevitable. $O$ sea, que el descenso de las catalanas podría haber sido mayor si las condiciones hubieran sido las del inicio del período.

Sexta. Se observa, además, un aumento de la descendencia de las generaciones barcelonesas nacidas después de $1926^{16}$, según Armand Sáez; pero no se puede esperar que este incremento, que comienza en niveles muy bajos (comparativamente a la descendencia de las mismas generaciones en el resto de España), alcance magnitudes muy superiores, dada la lentitud e inercia de los fenómenos demográficos.

Séptima. Las variaciones de la fecundidad de las catalanas residentes en Barcelona resulta limitada. El descenso del segundo quinquenio ha impedido que se aprovechara una situación estructural más favorable que antes.

En sintesis, la reducción de la fecundidad de las mujeres catalanas es, sobre todo, producto de las nuevas generaciones. Estos efectivos proceden de madres de dos orígenes; son hijas de catalanas y de migrantes. Por eso, su número resulta superior que en anteriores fechas. No se podrá decir de estas mujeres, al menos consideradas globalmente, que tienen una fecundidad elevada, sino lo contrario. Se podría presumir, aunque ello signifique una hipótesis que no se puede contrastar, que las hijas de las migrantes no siguen las pautas de fecundidad de origen de sus madres. Si el cambio, como considera $\mathrm{N}$. Ryder, es fundamentalmente generacional, en este caso se podría haber realizado en el menor plazo previsto ${ }^{17}$.

\section{LA COMUNIDAD MIGRANTE}

\section{Características generales}

Se considera comunidad migrante a las mujeres de 15 a 49 años que han nacido en el resto de Comunidades Autónomas de España, excepto Cataluña.

Los nacimientos de las mujeres migrantes presentan mayores alteraciones que la media de la ciudad. Sus incrementos, en números índices, son mayores y su descenso muy marcado, ya que se sitúa por debajo de la mitad del inicial,

${ }^{16}$ Armand SÁEz, La población de Barcelona, Ayuntamiento de Barcelona, 1971.

17 Norman Ryder, «Fertility and Family Structure», Population Bulletin of the United Nations, núm. 15, Nueva York, UN, 1983. 
en 1984. Existe, pues, una concentración de calendario en el primer quinquenio del 70. El descenso posterior es mayor que el de las catalanas, tanto si consideramos el año base como si consideramos el máximo de nacimientos del año 1974. Esta concentración puede ser tanto una recuperación de un atraso como un adelanto, teniendo en cuenta los bajos niveles posteriores (cuadro IV).

\section{CUADRO IV}

Nacimientos en Barcelona-ciudad de madres del resto de España: seis comunidades más numerosas y total

\begin{tabular}{|c|c|c|c|c|c|c|c|c|c|c|}
\hline & \multirow{2}{*}{\multicolumn{3}{|c|}{$A \tilde{n} o s$}} & \multirow{2}{*}{$\begin{array}{l}\text { Total } \\
\text { España } \\
\text { sin Cat. }\end{array}$} & \multicolumn{2}{|r|}{ LUGAR } & DE ORIGE & \multicolumn{2}{|c|}{ N DE LA MADRE } & \multirow[b]{2}{*}{ Galicia } \\
\hline & & & & & Andal. & Aragón & Cast. N. & Cast. V. & Extrem. & \\
\hline 1970 & $\ldots$ & $\ldots$ & & 100 & 100 & 100 & 100 & 100 & 100 & 100 \\
\hline 1971 & $\ldots$ & $\ldots$ & $\ldots$ & 110 & 112 & 98 & 113 & 108 & 115 & 110 \\
\hline 1972 & $\ldots$ & $\ldots$ & $\ldots$ & 117 & 111 & 100 & 106 & 108 & 118 & 119 \\
\hline 1973 & . ... & $\ldots$ & $\ldots$ & 118 & 111 & 96 & 107 & 110 & 125 & 116 \\
\hline 1974 & $\ldots$ & $\ldots$ & $\ldots$ & 123 & 111 & 98 & 105 & 126 & 125 & 121 \\
\hline 1975 & $\ldots$ & $\ldots$ & $\ldots$ & 111 & 114 & 84 & 114 & 87 & 125 & 126 \\
\hline 1976 & $\ldots$ & $\ldots$ & $\ldots$ & & & & & & & \\
\hline 1977 & $\ldots$ & $\ldots$ & $\ldots$ & 96 & 91 & 79 & 81 & 92 & 110 & .104 \\
\hline 1978 & $\ldots$ & $\ldots$ & $\ldots$ & 108 & 70 & 57 & 66 & 75 & 83 & 85 \\
\hline 1979 & $\ldots$ & $\ldots$ & $\ldots$ & 80 & 68 & 70 & 72 & 91 & 76 & 87 \\
\hline 1980 & $\ldots$ & $\ldots$ & $\ldots$ & 80 & 73 & 51 & 68 & 67 & 93 & 74 \\
\hline 1981 & $\ldots$ & $\ldots$ & $\ldots$ & 86 & 48 & 39 & 45 & 45 & 60 & 58 \\
\hline 1982 & $\ldots$ & $\ldots$ & $\ldots$ & 67 & 53 & 43 & 49 & 46 & 62 & 57 \\
\hline 1983 & $\ldots$ & $\ldots$ & $\ldots$ & 55 & 48 & 43 & 46 & 49 & 61 & 58 \\
\hline 1984 & ... & $\ldots$ & $\ldots$ & 49 & 44 & 37 & & & 52 & 53 \\
\hline
\end{tabular}

Elaboración propia.

FUENTES: Idem cuadro I.

Si la recuperación es cierta, ello explicaría el descenso posterior - sin necesidad de hablar de adelanto-, puesto que los nacimientos coinciden con una entrada de migrantes, y el posterior descenso correspondería a una más larga permanencia o a un flujo negativo, dado que las parejas más móviles son las más jóvenes y, por tanto, las que están, sobre todo, en edades más fecundas. La migración, como hecho fundamental en la historia de vida de las personas que la efectúan, podría afectar la intencionalidad o las decisiones respecto al número y momento de tener los hijos (gráfico adjunto).

Las comunidades más representadas en Barcelona ${ }^{18}$ - Andalucía, Aragón,

18 En la investigación se ha dividido al conjunto de migrantes en dos grupos, según su representatividad en la muestra realizada. Las seis comunidades mencionadas corresponden al grupo 1. Las comunidades con escasos efectivos - en alguno o en la mayoría de los grupos de edad- configuran el grupo 2. En esta exposición global de las migrantes no se estima necesario describir en profundidad cada uno de los grupos. 
Evolución general de los nacimientos según naturaleza de la madre: números indices, año base 1970

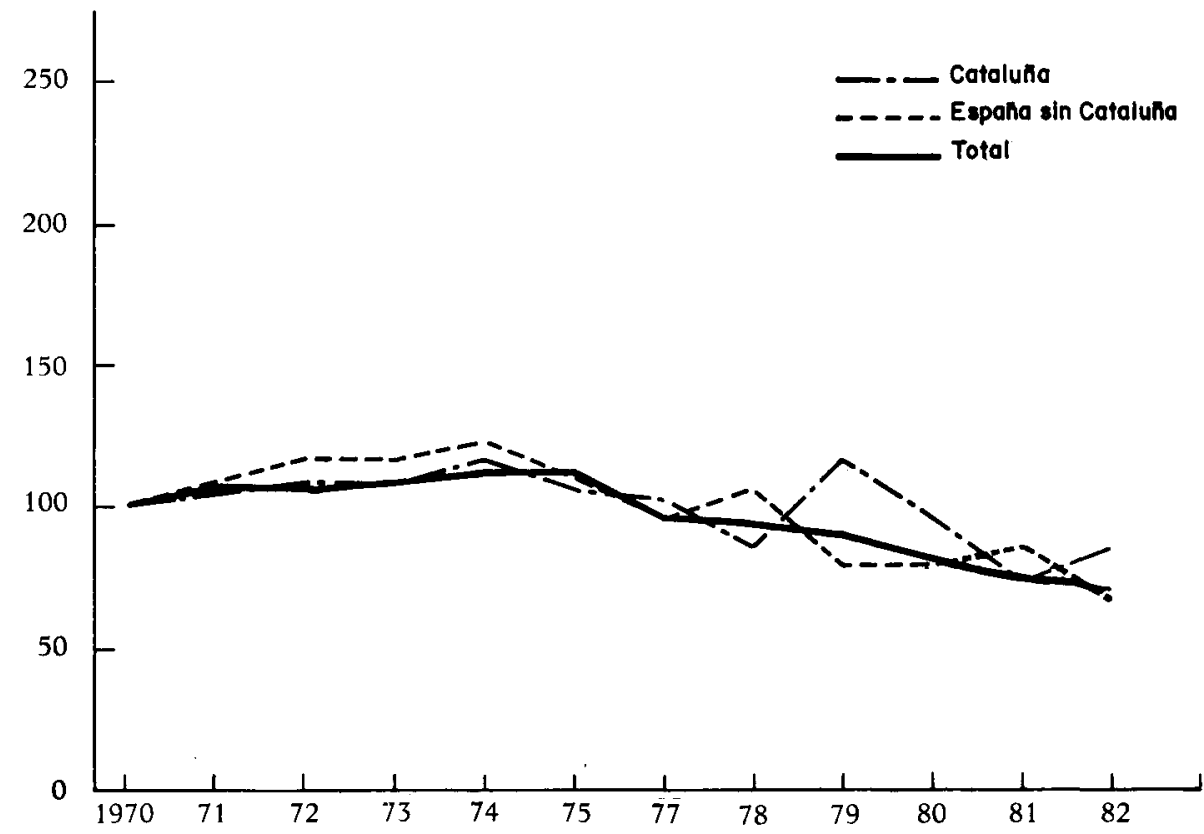

Castilla la Nueva, Castilla la Vieja, Extremadura y Galicia- presentan una evolución de los nacimientos similar al conjunto, puesto que sus aportes, globalmente considerados, determinan las tendencias generales, dado su peso cuantitativo.

Las mujeres correspondientes a las comunidades con menos efectivos en Barcelona también han limitado sus nacimientos, aunque en mucha mayor medida que las anteriores. Las razones podrían bien ser otras. En comunidades que en el pasado tuvieron mayor peso y representación en Barcelona, como es el caso de Valencia y Murcia, la pérdida de efectivos tiene efectos directos sobre el total de nacimientos. El efecto indirecto estaría producido por la probabilidad de un regreso, lo que podría retrasar el calendario de estas mujeres. Las comunidades insulares, Baleares y Canarias, se encuentran por debajo de la media de esta subpoblación - migrantes- y su descenso es muy acuciado.

Estas comunidades no sólo están más condicionadas que las de mayor representación por la pérdida de sus efectivos, sino que también podrían sentir, de manera diferente, las intencionalidades de retorno o emigración, dado su pequeño tamaño y la partida de muchos de sus componentes, lo que podría cuestionar su estancia. Las migraciones de retorno para estas comunidades 
podrían incrementarse, en el futuro, relativamente más que en las grandes. Pero desde hoy observaríamos las consecuencias en los niveles de nacimientos.

La baja de los nacimientos coincide con las comunidades que pierden peso, y los aumentos coinciden, también, con las que aumentan sus efectivos, y no solamente su peso relativo - como son las mujeres nacidas en las plazas de Africa- La reciente llegada de estas últimas podría explicar los únicos incrementos registrados en la segunda parte de la década del 70 y el único caso - junto con los extranjeros- en que aumentan tanto sus efectivos como los nacimientos absolutos, en 1984 -respecto del inicio de 1970-.

Dado el incremento relativo de las mujeres catalanas en los nacimientos ilegítimos, las migrantes descienden en su aporte al total. Sin embargo, al final del período su porcentaje todavía supera el de las catalanas.

Las tasas de fecundidad global de la comunidad migrante son del orden del doble que las correspondientes a las no-móviles. Registran un aumento en 1975-77, respecto de 1970-71, y un descenso posterior en 1980-81, que las sitúa por debajo de la tasa inicial. Sin embargo, las variaciones por comunidades son de mayor cuantía que las medias catalanas y migrantes. Las comunidades con más efectivos son las que tienen mayores tasas globales, mientras que las que poseen escasa cantidad de mujeres - salvo Canarias- son todas menores que la media. Las comunidades menos representadas en Barcelona poseen, en general, tasas muy próximas a la catalana - tanto superiores como inferiores, dependiendo de la fecha que se considere-. En cambio, al optar por comparar la tasa media para todo el período, ninguna comunidad de pocos efectivos presenta niveles superiores a la media del conjunto de migrantes, $y$ en tres casos resultan, además, menores que la catalana (cuadro II).

\section{En sintesis:}

Primero. El hecho de ser migrante no lleva aparejado tasas de fecundidad global superiores al conjunto de no-migrantes.

Segundo. Son las comunidades con más efectivos las que tienen una fecundidad superior y más aportan al hecho de una fecundidad diferencial, medida transversalmente.

Tercero. Las comunidades menos representadas en Barcelona poseen tasas vecinas a la catalana.

Cuarto. El aumento o disminución de los efectivos padronales coincide, sincrónicamente, con incrementos y disminuciones de las tasas globales.

Por ello, insistimos en que la proximidad de una migración puede alterar el calendario de estas mujeres; antes de realizarla, retrasándolo, y después del traslado, recuperando el atraso. 


\section{Nupcialidad y migración}

Con frecuencia se establecen relaciones directas entre la migración y otros fenómenos demográficos o sociales, olvidando los efectos indirectos y temporales que podrían inducir. Una formulación corriente en demografía es que los migrantes se casan más por tener acceso a dos mercados matrimoniales, en vez de sólo uno. Pero, a pesar de las probabilidades estadísticas, en general, las personas tienen comportamientos diferentes de los términos estrictamente matemáticos que se les atribuyen. La nupcialidad es uno de ellos. La homogamia, tanto social como geográfica o cultural, desmiente la libre elección inherente a la probabilidad de casarse con cualquiera del conjunto de la población.

Es cierto que las migrantes están más casadas, globalmente, que las locales, pero no es cierto que esto ocurra al interior de cada una de las comunidades; por lo que quedaría desmentida la anterior formulación, ya que no se verifica para todos los subgrupos.

Igual que señalara Zimmer, en su polémica con Westoff ${ }^{19}$, no debemos considerar solamente un grupo de referencia, en este caso la sociedad de destino, puesto que la sociedad de partida constituye el segundo marco de comparación obligado. Para poder afirmar que las migrantes se casan más, las dos comparaciones son necesarias: saber si se casan más que las mujeres no-móviles del sitio de partida y de destino.

Además, debemos considerar que las migrantes de las últimas décadas podrían corresponder relativamente más a familias y parejas constituidas que antes - que migraban más personas solas-. Los casamientos, en ese caso, estarían decididos y realizados en la sociedad de partida. Dicho de otro modo, una migración diferencial por estado civil, donde las casadas fueran mayoritarias y tuvieran mayor probabilidad de migrar que las solteras, tendría consecuencias en las proporciones de solteras de las migrantes en destino.

Las proporciones correspondientes a las seis comunidades más representadas en Barcelona resultan más reducidas que las del total de migrantes, salvo el primer grupo de $15-19$ años.

Las proporciones de las comunidades con menos efectivos presentan muchas oscilaciones, debido a lo reducido de su número en ciertos grupos de edad. En términos generales, tendrían mayor precocidad nupcial que la media de migrantes, pero mayor soltería en los grupos centrales y finales. De todas maneras, las situaciones resultan muy heterogéneas. Murcia y Valencia presentan proporciones en general reducidas, frente a Navarra y Euskadi, que las tienen elevadas.

Si comparamos estas proporciones de las residentes en Barcelona con las respectivas de origen, se observa que:

19 B. G. Zimmer, «The Impact of Social Mobility on Fertility: A Reconsideration», Population Studies, vol. 34, núm. 1, marzo 1980. 
- Del grupo de seis comunidades, sólo en cuatro las migrantes se casan más a partir de los 30 años. Antes de esa edad, la precocidad nupcial es mayor en origen que en destino ${ }^{20}$.

- En el grupo de comunidades menos representadas, las mujeres en origen tienen menores proporciones en los primeros grupos de edad. A partir de los 30 años, la soltería es mayor para las migrantes. $\mathrm{Si}$ estas mujeres no se han casado en origen y migran relativamente tarde, la frecuentación de un nuevo mercado matrimonial no parece aumentar sus posibilidades de contraer matrimonio, ya que permanecen solteras, en mayor proporción que en origen.

En sintesis, las proporciones resultan menores en el caso de las comunidades con mayor representación en la ciudad y cuanto mayores sean sus efectivos en las primeras edades.

En consecuencia, la migración estaría relacionada con un comportamiento diferenciado frente a la nupcialidad. Las migrantes no se casarían ni como en origen ni como en destino. Las mayores variaciones se registran en los grupos extremos. En las primeras edades, la migración podría precipitar, adelantar o anteceder a una boda. Si consideramos la intensidad del fenómeno - medida a partir de la soltería definitiva-, las mujeres que vinieron solteras permanecieron en ese estado civil en mayor proporción que las que quedaron en origen. En una palabra, dado que las proporciones de solteras de las migrantes no se alteran solamente por la nupcialidad en destino, sino también por la entrada en el denominador de mayor número de mujeres casadas, estas proporciones podrían variar, fundamentalmente, por las características o tipo de migraciones - solas, casadas, en familia con hijos-. En el pasado, las mujeres migraban más solas y solteras. En la actualidad, las migraciones corresponden, más que antes, a familias constituidas. Entonces, la migración no influiría tanto en la nupcialidad, sino que existiría una migración diferencial por estado civil, en las distintas épocas; lo que descalificaría la formulación de los dos mercados matrimoniales.

También debemos considerar un hecho complementario. Hasta ahora hemos comparado a las migrantes en origen y en destino y nos hemos limitado a las migrantes que entran, pero también debemos considerar las que salen. $\mathrm{Si}$ las migrantes que se van fueran mayoritariamente solteras, dado que su movilidad es mayor que la de una familia de varios miembros, esto también contribuiría a un descenso en la proporción de solteras. O sea, que si las casadas tuvieran una probabilidad mayor de migrar que las solteras en origen, las solteras, residentes en una comunidad diferente de la de nacimiento, podrían tener una mayor probabilidad de movimientos espaciales múltiples.

${ }^{20}$ Nota: Aragón y Castilla-León quedan excluidas de esta formulación, ya que en estas comunidades sucede lo contrario. 


\section{La estructura}

La proporción de las mujeres migrantes respecto de las catalanas de origen va reduciéndose continuamente. Además, como sus hijas han nacido mayoritariamente en Cataluña, figuran en la proporción de mujeres catalanas (cuadro V).

Lamentablemente, hasta ahora, en España no se ha podido medir la descendencia alcanzada antes de la migración. En Francia, por ejemplo, se encontró que las mujeres migrantes tenían, como media, un hijo menos que las no-móviles a edades tempranas.

En nuestro caso se puede estimar que si las migrantes hubieran traído hijos, lógicamente nacidos en origen o en otros sitios de migración anteriores a su residencia en Cataluña, los efectivos de la base de la pirámide catalana cuyo origen no fuera Cataluña serían mucho más grandes. La base de las pirámides catalanas de 1975 y 1981 es, casi exclusivamente y comparativamente al resto de las edades, de origen catalán.

La comunidad de migrantes varía su composición por origen a partir de 1975, al descender su proporción respecto al total de mujeres de la ciudad, pero sobre todo en 1981. Son las comunidades con más efectivos, especialmente Andalucía y Aragón, las que disminuyen respecto al total de migrantes. El aumento relativo de otras comunidades no implica un aumento absoluto de efectivos, sino una pérdida menor que otras o un estancamiento. Los efectivos de 1981 del conjunto de comunidades migrantes representan el 85 por 100 de los de 1970 . El descenso se registra en todos los grupos

\section{CUADRO V}

Estructura de la población femenina de $15-49$ años residente en Barcelona y cuyo lugar de nacimiento es el resto de España, en 1970, 1975 y 1981

$A \tilde{n} \circ s$

\begin{tabular}{|c|c|c|c|}
\hline & & & \\
\hline Grupos de edad & 1970 & 1975 & 1981 \\
\hline$\ldots \ldots \ldots \ldots$ & 19.874 & 13.806 & 10.756 \\
\hline$\ldots \ldots \ldots \ldots \ldots \ldots$ & 30.427 & 23.337 & 17.629 \\
\hline 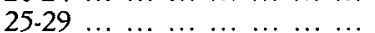 & 31.274 & 31.575 & 25.041 \\
\hline $\begin{array}{llllllll}30-34 & \ldots & \ldots & \ldots & \ldots & \ldots & \ldots & \ldots\end{array}$ & 30.107 & 31.270 & 28.862 \\
\hline$\ldots \ldots \ldots \ldots \ldots$ & 32.960 & 29.408 & 28.931 \\
\hline 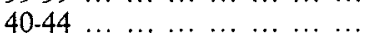 & 33.192 & 32.589 & 28.676 \\
\hline $\begin{array}{llllllll}45-49 & \ldots & \ldots & \ldots & \ldots & \ldots & \ldots & \ldots\end{array}$ & 31.776 & 32.637 & 32.183 \\
\hline Total ... ....... & 209.610 & 194.622 & 172.078 \\
\hline
\end{tabular}

Fuentes: Censos y Padrones de 1970, 1975 y 1981. 
de edades, salvo en el último, por un efecto «generación». Este descenso se explica por varias razones, a saber:

- Por un lado, los efectivos que avanzan en edad son menores que los anteriores.

- Las migraciones de entrada resultan insuficientes para llenar estos vacíos y sólo se producen en edades tempranas, hasta 29 años.

- A fa tir de los 30 años, las migraciones de salida son importantes y contribuyen a menguar aún más los efectivos de mujeres.

La distribución de las mujeres en edad fecunda también se altera durante el período, en sentido inverso al observado para las catalanas, o sea, la estructura tiende a ser cada vez más desfavorable a la fecundidad. Pero se mantiene más favorable que la media de la ciudad en 1970 y 1975.

O sea, que, en el caso de las migrantes, coinciden la mayor fecundidad con una estructura favorable y la menor fecundidad - global- con una estructura más envejecida. La elevada fecundidad del inicio y la posterior caída podrían ser explicadas, parcialmente, por la distribución de las mujeres en los distintos grupos de edad.

En sintesis, los factores que han contribuido a una fecundidad elevada al inicio y a una baja posterior son:

- Positivos, o favorables a la fecundidad:

- La entrada de migrantes en el período 70-75, superior a la salida.

- Una menor proporción de solteras que las catalanas, pero con grandes diferencias entre las distintas comunidades.

- Una estructura más favorable que la media de la ciudad, en 1970 y 1975.

- Negativos, o desfavorables a la fecundidad:

- Saldos negativos entre 1975 y 1981, lo que implica mayores salidas que entradas, pero un movimiento todavía importante.

- Descienden los efectivos absolutos de las mujeres en edad fecunda.

- La distribución resulta cada vez más envejecida debido al menor número en las generaciones que entran y avanzan en edad, a los mayores efectivos que salen y están en los últimos grupos y a las insuficientes entradas de migrantes, que resultan muy próximas a las salidas --salvo en el primer grupo de edad- La estructura resulta más desfavorable en 1981 que en 1970, y más desfavorable que la media de la ciudad para la última fecha (1981).

Como balance final se resumen las características de la fecundidad de los migrantes, destacando: 
Primera. El hecho de ser migrante no implica, necesariamente, una fecundidad global superior.

Segunda. Las variaciones y fluctuaciones de la fecundidad de las migrantes son mayores que las de las catalanas. Esto implicaría calendarios transversales en continuos cambios y que siguen la tendencia de los saldos migratorios.

Tercera. La división propuesta en dos grandes grupos de comunidades, según su peso cuantitativo, implica, además, diferencias intrínsecas, ya que existe cierta homogeneidad al interior de cada grupo y ambos contrastan en sus tendencias generales.

Cuarta. Respecto de la nupcialidad podríamos señalar que las proporciones de solteras resultaron diferentes, tanto en origen como en destino. Este hecho no significa que la migración influiría directamente en la nupcialidad, sino que podrían producirse migraciones diferenciales por estado civil. Será, pues, la probabilidad de migrar la que varíe según el estado civil de la persona. En el pasado, las migraciones podían tener una mayor proporción de solteras que en el presente. Las últimas generaciones pueden desplazarse más en parejas o grupos familiares que en forma individual. La mayor movilidad de las solteras puede conducirlas a multiplicar sus movimientos espaciales, por lo que los retornos o nuevas migraciones (en este caso de salida de la ciudad) pueden ser mayores. Además, la migración podría tener en origen un efecto inductor del matrimonio, al anticipar o preceder las bodas.

Quinta.-La ciudad de Barcelona podría tener un efecto negativo para la nupcialidad — de la misma manera que se habla del efecto de París sobre la reducción de la fecundidad-, que se haría notar: por un lado, en ciertos retrasos en el matrimonio, observables en la mayoría de los casos; por otro, sobre las catalanas en todas las edades y sobre las migrantes en las edades más avanzadas, provocando solterías definitivas relativamente elevadas.

\section{CONSIDERACIONES FINALES}

Para concluir se destacarán algunas particularidades observadas en el estudio de las mujeres migrantes:

1. Existe una cierta relación entre los efectivos de cada comunidad y otras variables que no es la meramente cuantitativa. Por ejemplo, el descenso de los efectivos, la distribución más desfavorable y los saldos migratorios negativos coinciden con el descenso de la fecundidad - en las catalanas sucedía lo contrario-. O sea, que las tendencias de la fecundidad coinciden con los indicadores estructurales y otras variables demográficas, lo que no significa que sus alteraciones puedan ser explicadas exclusivamente por estos factores. 
2. ${ }^{a} \quad$ En el caso de Barcelona no existe una tendencia del grupo minoritario por ser mayoritario ${ }^{21}$, al menos a través de su fecundidad, pero tampoco a través de los saldos migratorios --puesto que las pequeñas comunidades también tienen saldos negativos, aunque no en la misma proporción que las grandes.

3. ${ }^{2}$ El descenso de las proporciones de solteras en las edades donde la nupcialidad es más frecuente es usualmente explicado en términos del «efecto edad», o sea, que descienden cuando la edad avanza porque las personas se van casando. Sin embargo, si esta explicación puede resultar válida para las grandes poblaciones, podría no ser la única o principal razón de la baja en las poblaciones migrantes. Evidentemente, las migrantes también se casan, pero sus proporciones no varían exclusivamente por el efecto edad, sino quizá por migraciones diferenciales por estado civil ${ }^{22}$.

4. ${ }^{\mathrm{a}}$ Existe, además, otro fenómeno que relaciona nupcialidad y migración. Las tasas de nupcialidad catalanas para 1978 por lugar de celebración y residencia son diferentes. Mientras que en Barcelona y Tarragona residen más parejas que las que eligen como lugar de celebración de la boda estas provincias, en Lérida y Gerona ocurre lo contrario. Dados los saldos migratorios positivos e importantes que han tenido Barcelona y Tarragona en los últimos años, podemos suponer o bien que la gente se casa primero y migra inmediatamente después de la boda, o bien que la gente vuelva al lugar de origen, sobre todo si es próximo, para casarse. Las dos explicaciones podrían resultar posibles, puesto que no son excluyentes, pero sólo la segunda explicaría los casos de Lérida y Gerona. O sea, que, por una parte, podemos afirmar que algunas migraciones son precedidas por bodas; por la otra, las bodas podrían, a su vez, provocar migraciones temporales para llevarlas a cabo.

Las características señaladas de la población catalana y migrante sirven para poner énfasis en el hecho de que los desplazamientos y cambios de residencia tienden a alterar los comportamientos de las personas que los realizan, más allá de las clásicas influencias señaladas de las sociedades de origen y destino. Si bien las diferencias entre las distintas Comunidades Autónomas de España, en cuanto a comportamientos demográficos, existen, los contrastes entre nuevas y más antiguas generaciones, así como el hecho migratorio, podrían dividir a la población estudiada en grupos diferenciables por sus particularidades.

${ }^{21}$ Sobre esta cuestión se puede consultar el artículo de Eun Sul LEE y Robert RoBERTS, «Ethnic Fertility Differentials in the south west: the Case of Mexican American Rexamined», Sociology and Social Research, vol. 65, núm. 2, Los Angeles (California), 1981 , en que se intenta rebatir tal formulación.

${ }_{22}^{22}$ Nota: El contraste de las proporciones de solteras, a partir del Padrón de 1975 y el último censo, en algunas provincias que tradicionalmente expulsaban población, puede dar más indicios. 